\title{
TEMPERED ULTRADISTRIBUTIONS AS BOUNDARY VALUES OF ANALYTIC FUNCTIONS
}

\author{
BY
}

\author{
R. S. PATHAK
}

\begin{abstract}
The spaces $S_{a_{k}}, S^{h_{q}}$ and $S_{a_{k}}^{h_{q}}$ were introduced by I. M. Gel'fand as a generalization of the test function spaces of type $S$; the elements of the corresponding dual spaces are called tempered ultradistributions. It is shown that a function which is analytic in a tubular radial domain and satisfies a certain nonpolynomial growth condition has a distributional boundary value in the weak topology of the tempered ultradistribution space $\left(S_{b_{k}}^{a}\right)^{\prime}$, which is the space of Fourier transforms of elements in $\left(S_{a_{k}}^{b_{q}}\right)^{\prime}$. This gives rise to a representation of the Fourier transform of an element $U \in\left(S_{a_{k}}^{b_{a}}\right)^{\prime}$ having support in a certain convex set as a weak limit of the analytic function. Converse results are also obtained. These generalized PaleyWiener-Schwartz theorems are established by means of a number of new lemmas concerning $S_{a_{k}}^{b_{q}}$ and its dual. Finally, in the appendix the equality $S_{a_{k}}^{b_{q}}=S_{a_{k}} \cap S^{b_{q}}$ is proved.
\end{abstract}

1. Introduction. Ultradistributions are generalizations of Schwartz distributions [23]. The general idea consists in taking smaller spaces of test functions than $\mathscr{D}(\Omega)$, $\Omega \subset \mathbf{R}^{n}$, in such a way that essential statements of the theory remain true (in a generalized form), while the dual spaces are enlarged. The ideas of generalizing distributions can be found in the works of Beurling [2], Björck [3], Ehrenpreis [11-13], Gel'fand and Shilov [14], Roumieu [21, 22] and Schwartz [23]. Beurling and Björck defined spaces of test functions by growth properties of their Fourier transforms, while Roumieu used classes of ultradifferentiable functions taken from classical analysis. Komatsu [16] gave a unified treatment of both theories and proved deep and important structure theorems for these ultradistributions. Some special types of ultradistributions have also been studied by Gel'fand and Shilov [14] under the heading "Spaces of type $S$ " and these are generalizations of the well-known tempered distributions.

A further generalization of the spaces of type $S$ was given by I. M. Gel'fand [14, Appendix 1]; see also [21, 22]. In terms of arbitrary sequences $\left\{a_{k}\right\}(k=0,1,2, \ldots)$ and $\left\{b_{q}\right\}(q=0,1,2, \ldots)$, he defined three spaces of test functions known as $S_{a_{k}}, S^{b_{q}}$ and $S_{a_{k}}^{b_{q}}$. Babenko [1] studied the space $S_{a_{k}}^{b_{q}}$ and proved a general nontriviality theorem. In this paper we study a number of properties of the space $S_{a_{k}}^{b_{a}}$. In the Appendix we prove the important result $S_{a_{k}}^{b_{q}}=S_{a_{k}} \cap S^{b_{q}}$. The dual space of $S_{a_{k}}^{b_{q}}$ is denoted by $\left(S_{a_{k}}^{b_{q}}\right)^{\prime}$. It contains the space $S^{\prime}$ of tempered distributions. Furthermore,

Received by the editors December 21, 1982 and, in revised form, May 12, 1983.

1980 Mathematics Subject Classification. Primary 46F05, 46F15, 46F20, 46E10, 81E05.

(C)1984 American Mathematical Society $0002-9947 / 84 \$ 1.00+\$ .25$ per page 
the Björck space $S_{a}^{\prime} \subset\left(S_{a_{k}}^{a_{a}}\right)^{\prime}$; see Remark 3.8. The elements of the dual space will be called tempered ultradistributions.

Distributional boundary value results concerning analytic functions which have slow growth are important in quantum field theory; in particular the boundary values can be interpreted as vacuum expectation values in a field theory. Constantinescu [8] has obtained results representing vacuum expectation values as the boundary values of functions analytic in a tube domain coresponding to the forward light cone. Carmichael and Milton [7] have generalized the distributional boundary value results of Constantinescu [8, Theorems 1,2] to functions analytic in tube domains corresponding to open convex cones. Using spaces of type $W$, Pathak [17] extended some of the results of Carmichael and Milton. In these field theory results the analytic functions are recoverable via the Fourier-Laplace transform of a distribution which has support in a certain set and is the inverse Fourier transform of the vacuum expectation value. These boundary value results are generalizations of the Paley-Wiener theorem. We also note that Ehrenpreis [13] has obtained similar results for elements in analytically uniform spaces of distributions.

The vacuum expectation values in the axiomatic quantum field theory of $\mathrm{A}$. S. Wightman [24] can have at most polynomial increase in momentum space. A. Jaffe [15] has extended the axioms in order to allow nonpolynomial increase in momentum space. The theory of Jaffe fields (strictly localizable fields) has been studied by means of ultradistribution theory by Jaffe [15], Constantinescu and Thalheimer [9], Constantinescu [8] and De Roever [19]. In this paper we study Fourier-Laplace transform of tempered ultradistributions as boundary values of analytic functions of nonpolynomial growth. We extend some of the results of Carmichael and Milton, De Roever and Constantinescu referred to above. The present work will be useful in the study of nonstrictly localizable fields which have greater than polynomial growth in momentum space.

2. Notation and definitions. The $n$-dimensional notation used here will be that of Schwartz [23]. We define

$$
\langle x, y\rangle \stackrel{\Delta}{=} x_{1} y_{1}+\cdots+x_{n} y_{n}, \quad x \in \mathbf{R}^{n}, y \in \mathbf{R}^{n},
$$

with a similar definition for $\langle x, z\rangle, x \in \mathbf{R}^{n}, z \in \mathbf{C}^{n}$. Let $\alpha$ be an $n$-tuple of nonnegative integers. We define $|\alpha| \stackrel{\Delta}{=} \alpha_{1}+\cdots+\alpha_{n}$ and $\alpha ! \stackrel{\Delta}{=} \alpha_{1} ! \cdots \alpha_{n} !$. The differential operator $D^{\alpha}$ is defined by $D^{\alpha} \stackrel{\Delta}{=} D_{1}^{\alpha_{1}} \cdots D_{n}^{\alpha_{n}}$, where $D_{j}=\partial / \partial t_{j}, j=$ $1, \ldots, n$. We put $D_{t}^{\alpha}$ to indicate differentiation with respect to $t \in \mathbf{R}^{n}$. We similarly define $x^{\alpha} \stackrel{\Delta}{=} x_{1}^{\alpha_{1}} \cdots x_{n}^{\alpha_{n}}$ and $z^{\alpha} \stackrel{\Delta}{=} z_{1}^{\alpha_{1}} \cdots z_{n}^{\alpha_{n}}, x \in \mathbf{R}^{n}$ and $z \in \mathbf{C}^{n}$. The norm in $\mathbf{R}^{n}$ and $\mathbf{C}^{n}$ will be denoted by \|\| .

The Fourier transform of an $L_{1}$-function $\phi(t)$ is defined by

$$
\hat{\phi}(x)=\mathscr{F}[\phi(t) ; x]=\int_{\mathbf{R}^{n}} \phi(t) \exp (i\langle x, t\rangle) d t
$$


The inverse Fourier transform of $\phi \in L^{1}$ is defined by

$$
\mathscr{F}^{-1}[\phi(t) ; x]=(2 \pi)^{-n} \int_{\mathbf{R}^{n}} \phi(t) \exp (-i\langle x, t\rangle) d t .
$$

We refer to [27] for definition and terminology concerning cones. $C \subset \mathbf{R}^{n}$ is a cone with vertex at zero if $y \in C$ implies $\lambda y \in C$ for all positive scalars $\lambda$. The intersection of $C$ with the unit sphere $\|y\|=1$ is called the projection of $C$ and is denoted by $\operatorname{pr} C$. Let $C^{\prime}$ be a cone such that $\operatorname{pr} \bar{C}^{\prime} \subset \operatorname{pr} C$; then $C^{\prime}$ will be called a compact subcone of $C$. The function

$$
\mu_{C}(t)=\sup _{y \in \operatorname{pr} C}(-\langle t, y\rangle)
$$

is the indicatrix of $C . O(C)$ will denote the convex envelope of $C$.

$$
C^{*}=\left\{t: \mu_{C}(t) \leqslant 0\right\}=\{t:\langle t, y\rangle \geqslant 0, y \in C\}
$$

is the dual cone of $C$. The number

$$
\rho_{C}=\sup _{t \in C_{*}} \frac{u_{O(C)}(t)}{u_{C}(t)}, \quad C_{*}=\mathbf{R}^{n} \backslash C^{*},
$$

characterizes the nonconvexity of $C$. A cone $C$ is convex if and only if $\rho_{C}=1$ [27, Lemma 2, p. 220]. $T^{C}$ will denote the set $\mathbf{R}^{n}+i C \subset \mathbf{C}^{n}$. If $C$ is open and connected, $T^{C}$ will be called the tubular radial domain, while if $C$ is only open $T^{C}$ will be referred to as a tubular cone. Examples of tubular radial domains are the octants

$$
G_{\delta}=\left\{z: \delta_{j} \operatorname{Im}\left(z_{j}\right) \geqslant 0, \delta=\left(\delta_{1}, \ldots, \delta_{n}\right), \delta_{j}= \pm 1, j=1, \ldots, n\right\}
$$

and the forward light cone

$$
\Gamma^{+}=\left\{z: \operatorname{Im}\left(z_{1}\right)>\left(\sum_{j=2}^{n} \operatorname{Im}^{2}\left(z_{j}\right)\right)^{1 / 2}\right\} .
$$

We refer to Schwartz [23], Gel'fand and Shilov [14] and Edwards [10] for various definitions and terminology concerning topological vector spaces and their dual spaces. Let $C$ be an open connected cone, and let $C^{\prime}$ be an arbitrary compact subcone of $C$. Let $f(z)$ be a function of $z=x+i y \in T^{C}$; let $U$ be a generalized function. By $f(z) \rightarrow U$ in the weak topology of the generalized function space as $y=\operatorname{Im}(z) \rightarrow 0, y \in C^{\prime} \subset C$, we mean $\langle f(z), \psi(x)\rangle \rightarrow\langle U, \psi\rangle$ as $y \rightarrow 0, y \in C^{\prime}$ $\subset C$, for each fixed element $\psi$ in the corresponding test function space. By $f(z) \rightarrow U$ in the strong topology of the generalized function space as $y=\operatorname{Im}(z) \rightarrow 0, y \in C^{\prime}$ $\subset C$, we mean $\langle f(z), \psi(x)\rangle \rightarrow\langle U, \psi\rangle$ as $y \rightarrow 0, y \in C^{\prime} \subset C$, where the convergence is uniform for $\psi$ on arbitrary bounded sets in the corresponding test function space. We then call $U$ the weak (resp. strong) distributional boundary value of $f(z)$, and we note that it is defined on the distinguished boundary of $T^{C},\{z=x+i y$ : $\left.x \in \mathbf{R}^{n}, y=(0, \ldots, 0)\right\}$, which is not necessarily the topological boundary of $T^{C}$. In this paper we assume all open connected cones are convex. 
3. Test function spaces and their duals. Let $\left\{a_{k}\right\}$ and $\left\{b_{k}\right\}, k=0,1,2, \ldots$, be arbitrary sequences of positive real numbers. We will sometimes impose the following conditions on these sequences:

(M.1) $a_{p}^{2} \leqslant a_{p-1} a_{p+1}, p=1,2, \ldots$;

(M.2) there are constants $K$ and $H$ such that

$$
a_{p} \leqslant K H^{p} \min _{0 \leqslant q \leqslant p} a_{q} a_{p-q}, \quad p=1,2, \ldots ;
$$

(M.3) there is a constant $L$ such that

$$
\sum_{q=p+1}^{\infty} \frac{a_{q-1}}{a_{q}} \leqslant L p \frac{a_{p}}{a_{p+1}}, \quad p=1,2, \ldots
$$

It is well known that the Gevrey sequences $\left\{(p !)^{\alpha}\right\}$ and $\left\{p^{p \alpha}\right\}$ satisfy the above conditions for $\alpha>1[16$, p. 26].

Definition 3.1. A sequence $\left\{a_{p}\right\}$ (or $\left\{b_{p}\right\}$ ) of positive real numbers is said belong to class $\mathfrak{M}$ if it satisfies (M.1)-(M.3).

Now we recall the definition and properties, due to Gel'fand [14], of the test function space $S_{a_{k}}^{b_{a}}$. It consists of all infinitely differentiable functions $\phi$ defined on $\mathbf{R}^{n}$ which satisfy the inequalities

$$
\left|x^{\alpha} D^{\beta} \phi(x)\right| \leqslant C A^{k} B^{q} a_{k} b_{q}
$$

where $|\alpha|=k=0,1,2, \ldots,|\beta|=q=0,1,2, \ldots$, and $A, B, C$ are certain positive constants.

For $a_{k}=k^{k \alpha}, b_{q}=q^{q \beta}(\alpha, \beta \geqslant 0)$ the space $S_{a_{k}}^{b_{q}}$ reduces to the space $S_{\alpha}^{\beta}[14, \mathrm{p}$. 168] which is a well-known generalization of the Schwartz space $\mathscr{S}$ of test functions of rapid descent [23].

Now we define $S_{a_{k}}^{b_{q}}$ as the union of countably normed spaces. Let $S_{a_{k} \cdot A}^{b_{q, A}}$ denote the set of functions $\phi$ on $\mathbf{R}^{n}$ which, for any $\delta, \rho>0$, satisfy the inequalities

$$
\begin{aligned}
& \left|x^{\alpha} \phi^{\beta}(x)\right| \leqslant C_{\delta \rho}(A+\delta)^{k}(B+\rho)^{q} a_{k} b_{q} \\
& \quad(|\alpha|=k=0,1,2, \ldots,|\beta|=q=0,1,2, \ldots) .
\end{aligned}
$$

The topology over the space $S_{a_{k}, A}^{b_{q, B}}$ is generated by the collection of norms

$$
\|\phi\|_{\delta \rho}=\sup _{x, k, q} \frac{\left|x^{\alpha} \phi^{\beta}(x)\right|}{(A+\delta)^{k}(B+\rho)^{q} a_{k} b_{q}} \quad\left(\delta, \rho=1, \frac{1}{2}, \ldots\right),
$$

where $|\alpha|=k=0,1,2, \ldots$ and $|\beta|=q=0,1,2, \ldots$ It can easily be seen that $S_{a_{k}}^{b_{q}}$ is a complete countably normed perfect space [14, pp. 179-184]. It is defined as the union of the countably normed spaces $S_{a_{k}, A}^{b_{a . B}}$ for all indices $A, B=1,2, \ldots$ A sequence $\left\{\phi_{\nu}\right\}$ of elements of $S_{a_{k}}^{b_{q}}$ converges to zero in $S_{a_{k}}^{b_{q}}$ if (i) all the $\phi_{\nu}(x)$ belong to the same $S_{a_{k}, A}^{b_{q}, A}$, and (ii) $\left\{\phi_{\nu}\right\}$ converges to zero in the topology of $S_{a_{k}, A}^{b_{q, B}}$; this is the case if $\left\{\phi_{\nu}\right\}$ converges correctly to zero and the inequality

$$
\left|x^{\alpha} D^{\beta} \phi_{\nu}(x)\right| \leqslant C(A+\delta)^{k}(B+\rho)^{q} a_{k} b_{q}
$$

is satisfied, where the constants $A, B$ and $C$ are independent of $\nu$. 
If the sequences $\left\{a_{k}\right\}$ and $\left\{b_{q}\right\}$ belong to the class $\mathfrak{M}$ then from [22] we know that the Fourier transform of $\phi(t) \in S_{a_{k}}^{b_{q}}$ is $\psi(x)=\mathscr{F}[\phi(t) ; x]=\hat{\phi} \in S_{b_{k}}^{a_{q}}$. The Fourier transform and its inverse are topological isomorphisms between $S_{a_{k}}^{b_{q}}$ and $S_{b_{k}}^{a_{q}}$ [22, Theorem 20]. Symbolically we write

$$
\mathscr{F}\left[S_{a_{k}}^{b_{q}}\right]=S_{b_{k}}^{a_{q}}
$$

The Fourier transform of an element $U \in\left(S_{a_{k}}^{b_{q}}\right)^{\prime}$ is defined to be the element $V$ such that the Parseval relation

$$
\langle V, \psi\rangle=(2 \pi)^{n}\langle U, \phi\rangle
$$

holds, where $\phi \in S_{a_{k}}^{b_{q}}, \psi=\hat{\phi} \in\left(S_{b_{k}}^{a_{q}}\right)$, and $V$ is the Fourier transform of $U$, denoted $V=\mathscr{F}[U]$. In fact the Fourier transform is an isomorphism from $\left(S_{b_{k}}^{a_{q}}\right)^{\prime}$ onto $\left(S_{a_{k}}^{b_{q}}\right)^{\prime}$.

Associated functions.

Definition 3.2. For each sequence $\left\{b_{k}\right\}$ of positive numbers we define its associated function $b(\rho)$ on $(0, \infty)$ by

$$
b(\rho)=\sup _{k} \log \left(\rho^{k} b_{0} / b_{k}\right) .
$$

If $b_{k}$ satisfies (M.1) then by Komatsu [16, Proposition 3.2, p. 49],

$$
b_{k}=b_{0} \sup _{\rho}\left(\rho^{k} / \exp b(\rho)\right) \text {. }
$$

Conditions (M.1)-(M.3) can be expressed in terms of the associated function $b(\rho)$ [16]. (M.3) requires that $\int_{1}^{\infty} b(\rho) / \rho^{2} d \rho<\infty$. A necessary condition for this inequality is that for each $\varepsilon>0$ there is a $K(\varepsilon)>0$ such that for $\rho>0$,

$$
b(\rho) \leqslant \varepsilon \rho+K(\varepsilon),
$$

but this is not sufficient.

Definition 3.3. An operator of the form

$$
P(D)=\sum_{|\alpha|=0}^{\infty} e_{\alpha} D^{\alpha}, \quad e_{\alpha} \in \mathrm{C},
$$

is called an ultradifferentiable operator if for every $L>0$ there exists a constant $Q$ such that

$$
\left|e_{\alpha}\right| \leqslant Q L^{|\alpha|} / b_{|\alpha|}, \quad|\alpha|=0,1,2, \ldots
$$

If $\left\{b_{q}\right\}$ satisfies (M.1) then by [16, p. 58] the entire function

$$
P(z)=\sum_{|\alpha|=0}^{\infty} e_{\alpha} z^{\alpha}, \quad z \in \mathbf{C}^{n},
$$

satisfies the inequality

$$
|P(z)| \leqslant C \exp b(L\|z\|) .
$$

LEMMA 3.4. If $\left\{b_{q}\right\}$ satisfies (M.2) then the infinite order differential operator (3.9) is

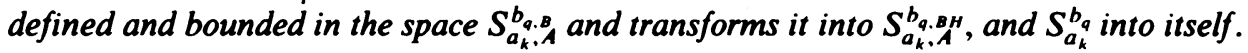


Proof. The proof is similar to [16, Theorem 2.12].

DEFinition 3.5. A positive increasing function $\eta$ on $[0, \infty)$, where $\eta(0)=0$ and $\eta(\rho) / \rho \rightarrow 0$ as $\rho \rightarrow \infty$, is called a subordinate function.

Lemma 3.6. Suppose $\left\{b_{q}\right\} \in \mathfrak{M}$. Then for every subordinate function $\eta$ there exists an ultradifferentiable operator $P_{\eta}(D)$ such that

$$
\left|P_{\eta}(i z)\right| \geqslant \exp b(\eta(\|z\|)), \quad\|y\|<1 .
$$

Proof. See [19, p. 44].

LEMMA 3.7. If $b_{k}$ satisfies (M.1) and (M.2) then the multiplication by $P($ ix) is $a$ continuous operation on $S_{a_{k}}^{b_{4}}$.

The proof is simple.

Another space with which we shall be concerned is $\mathscr{E}_{b_{4}}$, defined as follows: Let $K$ be a regular compact set in $\mathbf{R}^{n}$ and let $h>0$. We denote by $\mathscr{E}_{h_{q}}(K)$ the space of all $\phi \in C^{\infty}(K)$ satisfying

$$
\left|D^{\beta} \phi\right| \leqslant L h^{q} b_{q}, \quad|\beta|=q=0,1,2, \ldots,
$$

for some constant $L \geqslant 0$, and by $\mathscr{D}_{h_{q}}$ the space of all $f \in C^{\infty}\left(\mathbf{R}^{n}\right)$ with support in $K$ satisfying (3.13) for some $L \geqslant 0$. Clearly $\mathscr{E}_{h_{q}}(K)$ and $\mathscr{D}_{h_{q}}$ are Banach spaces with norm

$$
\|\phi\|=\sup _{\substack{x \in K \\ q}} \frac{\left|D^{\beta} \phi(x)\right|}{h^{q} b_{q}} .
$$

$\mathscr{D}_{h_{4}}$ may be looked upon as a closed subspace of $\mathscr{E}_{h_{4}}(K)$. The corresponding dual spaces are $\mathscr{D}_{b_{q}}^{\prime}$ and $\mathscr{E}_{b_{q}}^{\prime}(K)$.

If $\left\{b_{q}\right\}$ satisfies (M.1) it can easily be proved that $\mathscr{D}_{b_{4}}$ is a dense subspace of $S_{a_{k}}^{h_{q}}$ and $\left(S_{a_{k}}^{b_{q}}\right)^{\prime}$ is a subspace of $\mathscr{D}_{b_{q}}^{\prime}$.

REMARK 3.8. A function $a(\rho)$ associated with the sequence $\left\{a_{k}\right\}$ satisfying (M.1) is defined by $a(\rho)=\sup _{k} \log \left(\rho^{k} a_{0} / a_{k}\right)$. It plays the role of the Björck indicatrix $\omega$ occurring in the definition of the space $S_{\omega}$ [3]. In terms of $a(\rho)$ the topology over $S_{a_{k}}^{h_{4}}$ is defined by means of the norms

$$
\|\phi\|=\sup _{x, q}\left|\frac{\exp (a(x)) \phi^{\beta}(x)}{B^{q} b_{q}}\right|, \quad|\beta|=q=0,1,2, \ldots
$$

From (3.14) one can easily see that $\mathscr{D}_{a} \subset S_{a_{k}}^{a_{q}} \subset S_{a}$, where $\mathscr{D}_{a}$ is the Beurling space [3]. Moreover, $\mathscr{D}_{a}$ is dense in $S_{a}$. Hence $S_{a}^{\prime} \subset\left(S_{a_{k}}^{a_{q}}\right)^{\prime}$.

4. Some preliminary lemmas. In this section we establish lemmas concerning

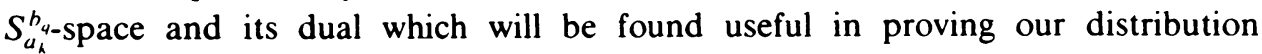
boundary value results.

Let \|\|$_{p}$ denote the $L_{p}$-norm; then the following lemma relates the sup norm $=$ \|\|$_{\infty}$ to the $L_{p}$-norm for $p=1,2$.

LemMa 4.1. Let $\phi \in S_{a_{k}}^{h_{q}}$ and let $\hat{\phi}(p)=\mathscr{F}[\phi(x)]$. Then

$$
\|\phi\|_{2}=\|\hat{\phi}\|_{2}, \quad\|\phi\|_{\infty} \leqslant(2 \pi)^{-n}\|\hat{\phi}\|_{1}
$$




$$
\begin{gathered}
\|\phi\|_{\infty} \leqslant(2 \sqrt{\pi})^{-n}\left(\|\phi\|_{2}+\|D \phi\|_{2}\right) ; \\
\left\|x^{\alpha} \phi^{\beta}(x)\right\|_{\infty} \\
\leqslant(2 \sqrt{\pi})^{-n}\left(\left\|x^{\alpha} \phi^{\beta}(x)\right\|_{2}+\left\|x^{\alpha} \phi^{\beta+1}(x)\right\|_{2}+\left\|\alpha x^{\alpha-1} \phi^{\beta}(x)\right\|_{2}\right) .
\end{gathered}
$$

Proof. For $\phi \in S_{a_{k}}^{b_{q}} \subset \mathscr{S}$, inequalities (4.1) are well known. Now,

$$
\begin{aligned}
\|\hat{\phi}\|_{1} & =\int_{\mathbf{R}^{n}}\left(1+p^{2}\right)^{1 / 2}|\hat{\phi}|\left(1+p^{2}\right)^{-1 / 2} d p \\
& \leqslant\left[\int_{\mathbf{R}^{n}}\left(1+p^{2}\right)^{-1} d p\right]^{1 / 2}\left[\int_{\mathbf{R}^{n}}\left(|\hat{\phi}|^{2}+|p \hat{\phi}|^{2}\right) d p\right]^{1 / 2} \\
& \leqslant \pi^{n / 2}\left[\|\hat{\phi}\|_{2}+\|p \hat{\phi}\|_{2}\right]
\end{aligned}
$$

so by (4.1),

$$
\|\phi\|_{\infty} \leqslant(2 \sqrt{\pi})^{-n}\left[\|\phi\|_{2}+\|D \phi\|_{2}\right] .
$$

(4.3) follows immediately from this relation.

LEMMA 4.2. Let $b_{k}$ satisfy (M.1). Suppose $\Phi \subseteq \mathscr{E}_{h_{4}}$ is such that for each $n$-tuple $\alpha$ of nonnegative integers, $\left\{D^{\alpha} \phi: \phi \in \Phi\right\}$ is uniformly bounded in $\mathscr{E}_{b_{4}}$ and $\Psi \subseteq S_{a_{k}}^{b_{4}}$ is bounded. Then $\Phi \Psi=\{\phi \psi: \phi \in \Phi ; \psi \in \Psi\}$ is bounded in $S_{a_{k}}^{b_{4}}$.

Proof. For $\phi \in \Phi$ and $\psi \in \Psi$, we have, by Leibnitz' rule,

$$
\begin{aligned}
\left|t^{\alpha} D^{\beta}(\phi \psi)(t)\right| & \leqslant \sum_{\gamma}\left(\begin{array}{c}
\beta \\
\gamma
\end{array}\right)\left|D^{\beta-\gamma} \phi\right|\left|t^{\alpha} D^{\gamma} \psi(t)\right| \\
& \leqslant \sum_{\gamma}\left(\begin{array}{c}
\beta \\
\gamma
\end{array}\right) C_{1} h^{|\beta-\gamma|} b_{|\beta-\gamma|} C_{2} A^{|\alpha|} B^{|\gamma|} a_{|\alpha|} b_{|\gamma|} .
\end{aligned}
$$

Therefore, in view of (M.1), we have

$$
\left|t^{\alpha} D^{\beta}(\phi \psi)(t)\right| \leqslant C_{3}(h+B)^{|\beta|} A^{|\alpha|} a_{|\alpha|} b_{|\beta|},
$$

where $C_{3}$ is another constant. This proves the lemma.

LEMMA 4.3. Let $C$ be an open convex cone. Let $a_{k}$ and $b_{q}$ satisfy (M.2) and (M.1), respectively, and $\left\{\psi_{y}(t): y \in C\right\} \subset \mathscr{E}_{b_{q}}$ such that for each n-tuple of nonnegative integers, $\left\{D_{t}^{\alpha}\left(\psi_{y}(t)\right): y \in C\right\}$ is uniformly bounded and $D_{t}^{\alpha}\left(\psi_{y}(t)\right) \rightarrow 0$ pointwise for $t \in \mathbf{R}^{n}$ as $y \rightarrow 0, y \in C$. Then for each $\phi \in S_{a_{k}}^{b_{a}}, \phi \psi_{y} \rightarrow 0$ in $S_{a_{k}}^{b_{q}}$ as $y \rightarrow 0, y \in C$.

Proof. Let $\phi$ be an arbitrary but fixed element of $S_{a_{a}}^{b_{4}}$. Then there exist $n$-tuples $A$ and $B$ such that $\phi \in S_{a_{k} \cdot A}^{b_{q}, A}$. Again by the generalized Leibnitz rule, we have

$$
\begin{aligned}
\left|t^{\alpha} D_{t}^{\beta}\left(\phi(t) \psi_{y}(t)\right)\right| & \leqslant \sum_{\gamma}\left(\begin{array}{c}
\beta \\
\gamma
\end{array}\right)\left|D_{t}^{\beta-\gamma} \psi_{y}(t)\right|\left|t^{\alpha} D_{t}^{\gamma} \phi(t)\right| \\
& \leqslant \sum_{\gamma}\left(\begin{array}{c}
\beta \\
\gamma
\end{array}\right) C_{1} h^{|\beta-\gamma|} b_{|\beta-\gamma|} C_{2}(A+\delta)^{|\alpha|}(B+\rho)^{|\gamma|} a_{|\alpha|} b_{|\gamma|} \\
& \leqslant C_{3}(A+\delta)^{|\alpha|} a_{|\alpha|} b_{|\beta|}(B+\rho+h)^{|\beta|}
\end{aligned}
$$


This inequality holds for all $t \in \mathbf{R}^{n}$ independently of $y \in \mathbf{C}^{n}$. It follows that the norms $\left\|\phi \psi_{y}\right\|_{\delta,(\rho+h)}$ are uniformly bounded independently of $y \in \mathbf{C}$.

Now we have

$$
\begin{aligned}
\left|t^{\alpha} D_{t}^{\beta}\left(\phi(t) \psi_{y}(t)\right)\right| \leqslant & \sum_{\gamma}\left(\begin{array}{c}
\beta \\
\gamma
\end{array}\right) C_{1} h^{|\beta-\gamma|} b_{|\beta-\gamma|} \\
& \cdot \prod_{i=1}^{n}\left(1+\left|t_{i}\right|\right)^{\alpha^{\prime}}\left|D_{t}^{\gamma} \phi(t)\right| \frac{\left|t^{\alpha}\right|}{\prod_{i=1}^{n}\left(1+\left|t_{i}\right|\right)^{\alpha_{i}^{\prime}}},
\end{aligned}
$$

where $\alpha_{i}^{\prime}=\alpha_{i}+2$. So that using (M.2) and the fact that

$$
\left(1+\left|t_{1}\right|\right)^{p} \cdots\left(1+\left|t_{n}\right|\right)^{p} \leqslant 2^{n p} \sup _{\alpha \leqslant p}\left|t_{11}^{\alpha} \cdots t_{n}^{\alpha_{n}}\right|
$$

we have

$$
\begin{aligned}
& \frac{\left|t^{\alpha} D_{i}^{\beta}\left(\phi(t) \psi_{y}(t)\right)\right|}{(A+\delta)^{k}(B+\rho)^{q} a_{k} b_{q}} \\
& \leqslant C_{1} \sum_{\gamma}\left(\begin{array}{c}
\beta \\
\gamma
\end{array}\right) h^{|\beta-\gamma|} b_{|\beta-\gamma|} 2^{n\left|\alpha^{\prime}\right|} \sup _{|l| \leqslant\left|\alpha^{\prime}\right|} \frac{\left|t^{\prime} D_{i}^{\gamma} \phi(t)\right|}{(A+\delta)^{|/|}(B+\rho)^{|\gamma|} a_{|l|} b_{|\gamma|}} \\
& \quad \prod_{i=1}^{n} \frac{\mid t_{i}^{\alpha_{i} \mid}}{\left(1+t_{i} \mid\right)^{\alpha_{i}^{\prime}}}(A+\delta)^{2} \frac{a_{\left|\alpha^{\prime}\right|}}{a_{k}} \frac{(B+\rho)^{|\gamma|}}{(B+\rho)^{q}} \frac{b_{|\gamma|}}{b_{q}} \\
& \leqslant C_{2} \sum_{\gamma}\left(\begin{array}{l}
\beta \\
\gamma
\end{array}\right) h^{|\beta-\gamma|} b_{|\beta-\gamma|} \frac{b_{|\gamma|}(B+\rho)^{|\gamma|}}{b_{q}(B+\rho)^{q}} \prod_{i=1}^{n} \frac{\mid t_{i}^{\alpha_{i} \mid}}{\left(1+\left|t_{i}\right|\right)^{\alpha_{i}^{\prime}}}\|\phi\|_{A, B, \delta, \rho} \\
& \leqslant C_{3} \frac{(B+\rho+h)^{q}}{(B+\rho)^{q}} \prod_{i=1}^{n} \frac{\mid t_{i}^{\alpha_{i} \mid}}{\left(1+\left|t_{i}\right|\right)^{\alpha_{i}^{\prime}}}\|\phi\|_{A, B, \delta, \rho .}
\end{aligned}
$$

This gives

$$
\left\|\phi \psi_{y}\right\|_{A_{1}, B_{1}, \delta, \rho} \leqslant C_{3}\|\phi\|_{A, B, \delta, \rho} \prod_{i=1}^{n} \frac{\left|t_{i}^{\alpha_{1}}\right|}{\left(1+\left|t_{i}\right|\right)^{\alpha_{i}^{\prime}}},
$$

where $A_{1}=A \max \left(1,2^{n} H\right), B_{1}=B+h$ and $\alpha^{\prime}=\alpha+2$. Notice that the right-hand side is Lebesgue integrable on $\mathbf{R}^{n}$ and the bound is independent of $y$. Further, from (4.4) we have

$$
\frac{\left|t^{\alpha} D_{t}^{\beta}\left(\phi(t) \psi_{y}(t)\right)\right|}{\left(A_{1}+\delta\right)^{k}\left(B_{1}+\rho\right)^{q} a_{k} b_{q}} \leqslant \sum_{\gamma}\left(\begin{array}{l}
\beta \\
\gamma
\end{array}\right) \frac{b_{|\gamma|}}{b_{q}}\left(\frac{B+\rho}{B_{1}+\rho}\right)^{|\gamma|}\left|D_{t}^{\beta-\gamma} \psi_{y}(t)\right| .
$$

The hypothesis that $D_{t}^{\alpha}\left(\psi_{y}(t)\right) \rightarrow 0$ pointwise in $\mathbf{R}^{n}$ as $y \rightarrow 0, y \in C$, for each $\alpha$, and (4.5) yield that

$$
\frac{\left|t^{\alpha} D_{t}^{\beta}\left(\phi(t) \psi_{y}(t)\right)\right|}{\left(A_{1}+\delta\right)^{k}\left(B_{1}+\rho\right)^{q} a_{k} b_{q}} \rightarrow 0
$$


pointwise on $\mathbf{R}^{n}$ as $y \rightarrow 0, y \in C$. Now, by the dominated convergence theorem, we have

$$
\lim _{\substack{y \rightarrow 0 \\ y \in C}} \int_{\mathbb{R}^{n}}\left|\frac{t^{\alpha} D_{t}^{\beta}\left(\phi(t) \psi_{y}(t)\right)}{\left(A_{1}+\delta\right)^{k}\left(B_{1}+\rho\right)^{q} a_{k} b_{q}}\right|^{2} d t=0 .
$$

Consequently, in view of (4.3) and (4.6), $\|\phi\|_{A_{1}, B_{1}, \delta . \rho} \rightarrow 0$ as $y \rightarrow 0, y \in C$. This completes the proof.

We note the following less restricted version of Lemma 4.3 which will be used in the sequel.

LEMMA 4.4. Let $a_{k}$ and $b_{q}$ satisfy (M.2) and (M.1), respectively. Let $C$ be an open convex cone and let $Q$ be an arbitrary but fixed positive real number. Let $\left\{\psi_{y}(t)\right.$ : $y \in C\} \subset \mathscr{E}_{b_{q}}$ such that for each n-tuple of nonnegative integers, $\left\{D_{t}^{q}\left(\psi_{y}(t)\right): y \in C\right.$, $|y| \leqslant q\}$ is uniformly bounded and $D_{t}^{q}\left(\psi_{y}(t)\right) \rightarrow 0$ pointwise for $t \in \mathbf{R}^{n}$ as $y \rightarrow 0$, $y \in C$. Then for each $\phi \in S_{a_{k}}^{b_{q}}, \phi \psi_{y} \rightarrow 0$ in $S_{a_{k}}^{b_{a}}$ as $y \rightarrow 0, y \in C$.

Let $b \geqslant 0$ be fixed. Let $\xi(\eta) \in \mathscr{E}_{b_{q}}, \eta \in \mathbf{R}$, such that $\xi(\eta)=1, \eta \geqslant-b, \xi(\eta)=0$, $\eta \leqslant(-b-\varepsilon), \varepsilon>0$ and fixed, and $0 \leqslant \xi(\eta) \leqslant 1$. Put

$$
\lambda(t)=\xi(\langle y, t\rangle) \quad\left(y \in C, t \in \mathbf{R}^{n}\right),
$$

where $C$ is an open convex cone. We have $\lambda(t) \in \mathscr{E}_{b_{q}}, t \in \mathbf{R}^{n}$ and $y \in C^{\prime} \Subset C$.

LEMMA 4.5. Let $a_{k}$ satisfy (M.2) and $b_{q}$ satisfy (M.1). Let $C$ be an open convex cone and $C^{\prime}$ a compact subcone of $C$. Let $\phi \in S_{a_{k}}^{b_{a}}$. Let $\lambda(t)$ be the function defined by (4.7), where $b \geqslant 0$ is arbitrary but fixed. Then

$$
\lambda(t) \exp (-\langle y, t\rangle) \phi(t) \rightarrow \lambda(t) \phi(t)
$$

in $S_{a_{k}}^{b_{a}}$ as $y \rightarrow 0, y \in C^{\prime} \Subset C$.

Proof. Let us set $\psi_{y}(t)=\lambda(t)(\exp (-\langle y, t\rangle)-1), y \in C^{\prime} \Subset C$, and use Lemma 4.4. Then we have

$$
\left|D_{t}^{\beta} \psi_{y}(t)\right|=\left|\sum_{\gamma}\left(\begin{array}{c}
\beta \\
\gamma
\end{array}\right) D_{t}^{\gamma} \lambda(t)\left((-y)^{\beta-\gamma} \exp (-\langle y, t\rangle)-D^{\beta-\gamma}(1)\right)\right| .
$$

Now $\exp (-\langle y, t\rangle) \rightarrow 1$ pointwise for $t \in \mathbf{R}^{n}$ as $y \rightarrow 0, y \in C$. Since $D^{\gamma} \lambda(t)$ is bounded on $\mathbf{R}^{n}$ for each $\gamma$, we have that the term in the sum in (4.8) which corresponds to $\gamma=\beta$ satisfies

$$
\lim _{\substack{y \rightarrow 0 \\ y \in C}}\left(D^{\beta} \lambda(t)\left(e^{-\langle y, t\rangle}-1\right)\right)=0
$$

pointwise for $t \in \mathbf{R}^{n}$. In order to obtain a similar convergence in the other terms in the sum (4.8), we recall that by the definition of $\lambda(t), D^{\gamma} \lambda(t)=0$ for all $\gamma \leqslant \beta$ and $t \in \mathbf{R}^{n}$ such that $\langle y, t\rangle \leqslant-b-\varepsilon, y \in C$. Thus $D_{t}^{\beta} \psi_{y}(t)=0$ if $\langle y, t\rangle \leqslant-b-\varepsilon$, $y \in C$, so it suffices to consider $D_{t}^{\beta} \psi_{y}(t)$ for $t \in \mathbf{R}^{n}$ such that $\langle y, t\rangle>-b-\varepsilon$, $y \in C$. For other terms in the sum corresponding to those $\gamma \neq \beta, D^{\beta-\gamma}(1)=0$, and 


$$
\begin{aligned}
& \text { for }\langle y, t\rangle>-b-\varepsilon, y \in C^{\prime} \text {, } \\
& \left|D^{\gamma} \lambda(t)(-y)^{\beta-\gamma} e^{-\langle y, t\rangle}\right| \leqslant L B^{|\gamma|} b_{|\gamma|} e^{(b+\varepsilon)}\left|y^{\beta-\gamma}\right|, \\
& \left|D_{t}^{\beta} \psi_{y}(t)\right| \leqslant L e^{(b+\varepsilon)} \sum_{\gamma}\left(\begin{array}{c}
\beta \\
\gamma
\end{array}\right) B^{|\gamma|} b_{|\gamma|}\left|y^{\beta-\gamma}\right| \\
& \leqslant L e^{(b+\varepsilon)} b_{q} \sum_{\gamma}\left(\begin{array}{l}
\beta \\
\gamma
\end{array}\right) B^{|\gamma|}\left(\frac{b_{0}}{b_{1}}\right)^{q-|\gamma|}(1+|y|)^{|\beta-\gamma|} \\
& \leqslant L e^{(b+\varepsilon)} b_{q}\left(\frac{b_{0}}{b_{1}}\right)^{q} \sum_{\gamma}\left(\begin{array}{l}
\beta \\
\gamma
\end{array}\right)\left(\frac{B b_{1}}{b_{0}}\right)^{|\gamma|}(|y|+1)^{|\beta-\gamma|} \\
& \leqslant L e^{(b+\varepsilon)} b_{q}\left(\frac{b_{0}}{b_{1}}\right)^{q}\left(\frac{B b_{1}}{b_{0}}+|y|+1\right)^{q} \\
& \leqslant L e^{(b+\varepsilon)}\left(B+\frac{b_{0}}{b_{1}}(|y|+1)\right)^{q} b_{q} \quad(q=|\beta|) .
\end{aligned}
$$

Thus $\psi_{y}(t) \in \mathscr{E}_{h_{u}}$ for $y \in C^{\prime} \Subset C$ and $D_{t}^{\beta} \psi_{y}(t)$ is uniformly bounded for $|y| \leqslant Q$, $y \in C$. We conclude from the previous lemma that

$$
\lambda(t)(\exp (-\langle y, t\rangle)-1) \phi(t) \rightarrow 0
$$

in $S_{a_{h}}^{h_{4}}$ as $y \rightarrow 0, y \in C^{\prime} \Subset C$, which proves the desired result.

LeMma 4.6. Assume $a_{k}$ and $b_{q}$ are as in Lemma 4.5. Let $C$ be an open convex cone and $\Psi$ a bounded set in $S_{a_{k}}^{b_{a}}$. Let $\lambda(t)$ be the function defined by (4.7), where $b \geqslant 0$ is arbitrary but fixed. Let

$$
\Phi=\{\lambda(t) \exp (-\langle y, t\rangle): y \in C,|y| \leqslant Q\} .
$$

where $Q$ is an arbitrary but fixed positive real number. Then $\Phi \Psi$ is a bounded set in $S_{a_{h}}^{h_{4}}$.

Proof. From the proof of Lemma $4.5, \Phi \in \mathscr{E}_{h_{q}}$ and $\left\{D_{t}^{\alpha}(\lambda(t) \exp (-\langle y, t\rangle))\right.$ : $y \in C,|y| \leqslant Q\}$ is uniformly bounded for each $n$-tuple $\alpha$ of nonnegative integers. By Lemma 4.2, $\Phi \Psi$ is a bounded set in $S_{a_{h}}^{b_{4}}$.

LemMA 4.7. Let $b_{q}$ satisfy (M.1). Let $U \in\left(S_{a_{k}}^{h_{4}}\right)^{\prime}$ and $\theta \in S_{a_{k}}^{h_{4}}$. Then $\theta U \in\left(S_{a_{k}}^{h_{4}}\right)^{\prime}$.

Proof. Let $\phi \in S_{a_{k}}^{h_{4}}$. Then

$$
\begin{aligned}
x^{\alpha} D^{\beta}(\theta \phi) & =\sum_{\gamma} x^{\alpha}\left(\begin{array}{c}
\beta \\
\gamma
\end{array}\right) D^{\gamma} \theta D^{\beta-\gamma} \phi \\
& \leqslant \sum_{\gamma}\left(\begin{array}{c}
\beta \\
\gamma
\end{array}\right) C_{1} A^{|\alpha|} B^{|\gamma|} a_{|\alpha|} b_{|\gamma|} \cdot C_{2} A_{1}^{0} B_{1}^{|\beta-\gamma|} a_{0} b_{|\beta-\gamma|} \\
& \leqslant C A^{|\alpha|} a_{0} a_{|\alpha|} \sum_{\gamma}\left(\begin{array}{c}
\beta \\
\gamma
\end{array}\right) B^{|\gamma|} B_{1}^{|\beta-\gamma|} b_{|\beta|} \\
& \leqslant C^{\prime} A^{|\alpha|} A_{|\alpha|} b_{|\beta|}\left(B+B_{1}\right)^{|\beta|}
\end{aligned}
$$


so $\theta \phi \in S_{a_{k}}^{b_{q}}$ for all $\phi \in S_{a_{k}}^{b_{q}}$. The boundedness of the derivatives of $\theta$ yields also that $\left\{\phi_{\nu}\right\} \rightarrow 0$ in $S_{a_{k}}^{b_{q}}$ implies $\left\{\theta \phi_{\nu}\right\} \rightarrow 0$ in $S_{a_{k}}^{b_{q}}$ for $\nu \rightarrow \infty$. Hence $\theta$ is a multiplicator in $\left(S_{a_{k}}^{b_{q}}\right)^{\prime}$ and $\theta U \in\left(S_{a_{k}}^{b_{q}}\right)^{\prime}$.

5. Paley-Wiener theorems for tempered ultradistributions. Let $C$ be an open, convex cone in $\mathbf{R}^{n}$ and let $h$ be a convex function on $C$, homogeneous of degree one. The pair $(h, C)$ determines uniquely a closed convex set $U(h, C)$, not containing a straight line, in $\mathbf{R}^{n}$ by

$$
U(h, C)=\{t:-\langle t, y\rangle \leqslant h(y), y \in C\} .
$$

Conversely, each closed, convex set $U$ in $\mathbf{R}^{n}$, which does not contain a straight line, uniquely determines an open, convex cone $C$ in $\mathbf{R}^{n}$ and a homogeneous, convex function $h$ on $C$ such that $U=U(h, C)$, according to (5.1); see [20].

We now define the space of analytic functions $H_{C}^{h}$ whose elements have distributional boundary values in the space $\left(S_{b_{q}}^{a_{k}}\right)^{\prime}$, the space of Fourier transforms of tempered ultradistributions. Let $C^{\prime}$ be an arbitrary compact subcone of the cone $C$. Let $N(0, m)$ denote a closed ball of the origin in $\mathbf{R}^{n}$ of radius $m$, where $m$ is an arbitrary positive real number. Consider the function $f(z)$ satisfying

$$
|f(z)| \leqslant K\left(C^{\prime}, m, L\right) \exp \{b(L\|z\|)+h(y)+\sigma\|y\|\} \quad\left(z \in T\left(C^{\prime}, m\right)\right),
$$

for all $L, \sigma>0$, where $b$ is the function defined by (3.6), $h(y)$ is the same as in (5.1),

$$
T\left(C^{\prime}, m\right)=\mathbf{R}^{n}+i\left(C^{\prime} \backslash\left(C^{\prime} \cap N(0, m)\right)\right),
$$

and $K\left(C^{\prime}, m, L\right)$ is a constant depending on $C^{\prime}, m$ and $L>0$. The set of all functions $f(z)$ analytic in $T\left(C^{\prime}, m\right)$ and satisfying (5.2) for all compact subcones $C^{\prime} \subset C$ and for arbitrary positive number $m$ is denoted by $H_{C}^{h}$.

In the following theorem, which may be regarded as the Paley-Wiener theorem for tempered ultradistributions, we show that functions in $H_{C}^{h}$ have distributional boundary values in $\left(S_{b_{k}}^{a_{k}}\right)^{\prime}$. In this theorem we shall be concerned with the cones possessing the following property.

Definition 5.1. An open convex cone $C$ is said to have property $\mathrm{P}$ if for each $\phi \in S_{a_{k}}^{b_{q}}$ there exist $n$-tuples $A=\left(A_{1}, \ldots, A_{n}\right)$ and $B=\left(B_{1}, \ldots, B_{n}\right)$ such that $A$, $B \in C$ and $\phi \in S_{a_{k}, A}^{b_{q}, A}$.

TheOREM 5.2. Suppose $\left\{a_{k}\right\} \in \mathfrak{M}$ and $\left\{b_{k}\right\} \in \mathfrak{M}$. Let $f(z) \in H_{C}^{h}$, and let $C$ be an open convex cone such that each compact subcone $C^{\prime} \subset C$ satisfies property $\mathrm{P}$. Then there exists a unique element $U \in\left(S_{a_{k}}^{b_{a}}\right)^{\prime}$ with $\operatorname{supp}(U) \subseteq U\left(h, C^{\prime}\right)=\{t:-\langle t, y\rangle \leqslant$ $\left.h(y), y \in C^{\prime}\right\}$ such that $f(z) \rightarrow \mathscr{F}[U] \in\left(S_{h_{k}}^{a_{a}}\right)^{\prime}$ in the weak topology of $\left(S_{h_{k}}^{\left.a_{q}\right)^{\prime}}\right.$ as $y=\operatorname{Im}(z) \rightarrow 0, y \in C^{\prime} \Subset C$.

Proof. Let $K(L)$ denote the constant $K\left(C^{\prime}, m, L\right)$ occurring in the inequality (5.2). We define a subordinate function $\eta(\rho)$ (cf. Definition 3.5) by

$$
b(\eta(\rho))=\inf _{L}\{b(L \rho)+\log (K(L) / K(1))\} ;
$$

then by [16, after Lemma 9.5] it follows that $\eta(\rho) / \rho \rightarrow 0$ as $\rho \rightarrow \infty$. So, from (5.2),

$$
|f(z)| \leqslant K\left(C^{\prime}, m, 1\right) \exp \{b(\eta(\|z\|))+\sigma(\|y\|)+h(y)\} .
$$


Now, in view of Lemma 3.6, there exists an ultradifferentiable operator $P(D)$ depending on $\eta^{\prime}$ such that

$$
P_{\eta^{\prime}}(i z) \geqslant \exp b\left(\eta^{\prime}(\|z\|)\right)>\exp b(\eta(\|z\|)) .
$$

Set $P(i z)=P_{\eta^{\prime}}(i z)$. Then choose $\eta^{\prime}$ such that

$$
g_{y}(t)=(2 \pi)^{-n} \int_{\mathbf{R}^{n}} \frac{f(z)}{P(i z)} \exp (-i\langle z, t\rangle) d x, \quad z \in T\left(C^{\prime}, m\right),
$$

exists finitely. Then $g_{y}(t)$ is a continuous function of $t$ and, by the Cauchy-Poincaré theorem [27, p. 198], it is independent of $y$. We therefore denote it by $g(t)$. From (5.5) we have $f(z) / P(i z) \in L^{1} \cap L^{2}$ as a function of $x=\operatorname{Re} z \in \mathbf{R}^{n}, y \in$ $C^{\prime} \backslash\left(C^{\prime} \cap N(0, m)\right)$. Thus by (5.5) and Plancherel theory $\exp (-\langle y, t\rangle) g(t) \in L^{2}$ as a function of $t \in \mathbf{R}^{n}$, and

$$
f(z) / P(i z)=\mathscr{F}[\exp (-\langle y, t\rangle) g(t): x] \quad\left(z \in T\left(C^{\prime}, m\right)\right),
$$

where the Fourier transform is in the $L_{2}$-sense. Here, Parseval's equation holds:

$$
(2 \pi)^{n} \int_{\mathbf{R}^{n}}|g(t)|^{2} e^{-2\langle y, t\rangle} d t=\int_{\mathbf{R}^{n}}\left|\frac{f(z)}{P(i z)}\right|^{2} d x .
$$

Let us show that $g(t) \in L^{2}$. In view of inequality (5.5), from (5.9), we obtain

$$
\begin{aligned}
(2 \pi)^{n} \int_{\mathbf{R}^{n}}|g(t)|^{2} e^{-2\langle y, t\rangle} d t \leqslant & K^{2} \exp \{2(h(y)+\sigma\|y\|)\} \\
& \cdot \int_{\mathbf{R}^{n}} \exp \left[b(\eta(\|z\|))-b\left(\eta^{\prime}(\|z\|)\right)\right] d x, \quad y \in C^{\prime} .
\end{aligned}
$$

From this and from Fatou's lemma [27, p. 12], we conclude that

$$
(2 \pi)^{n} \int_{|t| \leqslant R}|g(t)|^{2} d t \leqslant \varlimsup_{\substack{y \rightarrow 0 \\ y \in C^{\prime}}} \int_{|t| \leqslant R}|g(t)|^{2} e^{-2\langle y, t\rangle} d t \leqslant K_{1}\left(C^{\prime}\right),
$$

for arbitrary $R>0$, so $g(t) \in L^{2}$.

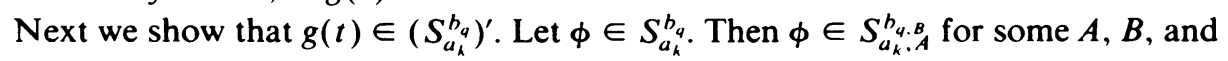

$$
\begin{aligned}
\int_{\mathbf{R}^{n}}|g(t) \phi(t)| d t= & \int_{|t| \leqslant 1} a_{0} b_{0}|g(t)| \frac{|\phi(t)|}{a_{0} b_{0}} d t \\
& +\int_{|t|>1}|t|^{-2}|g(t)| \frac{|t|^{2}|\phi(t)|}{a_{2} b_{0}(A+\delta)^{2}} d t \cdot a_{2} b_{0}(A+\delta)^{2} \\
& \leqslant C_{1}\|\phi\|\left(\int_{|t| \leqslant 1}|g(t)|^{2} d t\right)^{1 / 2}+C_{2}\|\phi\|\left(\int_{|t|>1}|g(t)|^{2} d t\right)^{1 / 2} \\
& <\infty .
\end{aligned}
$$

Therefore, as a regular generalized function, $g(t) \in\left(S_{a_{k}}^{b_{a}}\right)^{\prime}$. Also, by Lemma 3.4, $U=P(-D) g(t) \in\left(S_{a_{k}}^{h_{q}}\right)^{\prime}$.

It remains to show that $g(t)=0$ for $t \in \mathbf{R}^{n} \backslash U\left(h, C^{\prime}\right)$. It will be sufficient to prove this result for a small neighbourhood $\left\|t-t_{0}\right\|<\eta$ of an arbitrary point $t_{0}$ in 
the domain $\mathbf{R}^{n} \backslash U\left(h, C^{\prime}\right)$. Then there exists a point $y_{0} \in C^{\prime}$ with $\left\|y_{0}\right\|=1$ such that $-\left\langle t_{0}, y_{0}\right\rangle>h\left(y_{0}\right)$. Let $\eta>0$ be so small that $-\left\langle t_{0}, y_{0}\right\rangle \geqslant h\left(y_{0}\right)+\eta$. Let $\lambda$ be an arbitrary positive number, and put $y=\lambda y_{0}$. Then

$$
\left\langle t_{0}, y\right\rangle=\lambda\left\langle t_{0}, y_{0}\right\rangle \leqslant-\lambda\left(h\left(y_{0}\right)+\eta\right) .
$$

Setting $C^{\prime}=\left\{y: y=\lambda y_{0}\right\}$ and using the fact that for $y \in C^{\prime},\|y\|=\lambda$, from (5.5), (5.7), (5.10) and (3.8), we have

$$
\begin{aligned}
\left|g\left(t_{0}\right)\right| & \leqslant K_{\varepsilon} \exp \left[-\lambda\left(h\left(y_{0}\right)+\eta\right)+\|y\| h(y /\|y\|)+\sigma \lambda+\varepsilon L \lambda\right] \\
& =K_{\varepsilon} \exp [-\lambda(\eta-\sigma-\varepsilon L)] .
\end{aligned}
$$

Choosing $\varepsilon$ and $\sigma$ such that $\sigma+\varepsilon L<\eta$ and letting $\lambda \rightarrow \infty$, we see that $g\left(t_{0}\right)=0$. Thus $\operatorname{supp}(g) \subseteq U\left(h, C^{\prime}\right)$, since $t_{0}$ is arbitrary and $U\left(h, C^{\prime}\right)$ is a closed set.

Next we must prove convergence. Since, as a regular generalized function, $\exp (-\langle y, t\rangle) g(t) \in\left(S_{a_{k}}^{b_{q}}\right)^{\prime}, y \in C^{\prime} \backslash\left(C^{\prime} \cap N(0, m)\right)$ and $f(z) / P(i z) \in L^{1} \cap L^{2}$ as a function of $x \in \mathbf{R}^{n},(5.8)$ holds as an equality in $\left(S_{a_{k}}^{b_{q}}\right)^{\prime}$. Let $\psi \in S_{b_{k}}^{a_{q}}$ and $\phi \in S_{a_{k}}^{b_{q}}$ such that $\psi=\mathscr{F}[\phi]$. Then from (5.8) and (3.5), we have

$$
\begin{aligned}
\langle f(z) / P(i z), \psi(x)\rangle=(2 \pi)^{n}\langle\exp (-\langle y, t\rangle) g(t), \phi(t)\rangle & \left(z \in T\left(C^{\prime}, m\right)\right)
\end{aligned}
$$

for $y=\operatorname{Im}(z)$ arbitrary but fixed in $C^{\prime} \backslash\left(C^{\prime} \backslash N(0, m)\right)$, where $m$ may be arbitrarily small and positive. Let $b=\sup _{y}|h(y)|, y \in C^{\prime}$. Since, $\operatorname{supp}(g) \subset U\left(h, C^{\prime}\right)$, we have

$$
\langle\exp (-\langle y, t\rangle) g(t), \phi(t)\rangle=\langle g(t), \lambda(t) \exp (-\langle y, t\rangle) \phi(t)\rangle,
$$

where $\lambda(t)$ is the same as defined by (4.7). Since $g(t) \in\left(S_{a_{k}}^{b_{q}}\right)^{\prime}$ and $\operatorname{supp}(g) \subset$ $U\left(h, C^{\prime}\right)$, from Lemma 4.5 we conclude that

$$
\begin{aligned}
\lim _{\substack{y \rightarrow 0 \\
y \in C^{\prime} \Subset C}}\langle g(t), \lambda(t) \exp (-\langle y, t\rangle) \phi(t)\rangle \\
=\langle g(t), \lambda(t) \phi(t)\rangle=\langle g(t), \phi(t)\rangle .
\end{aligned}
$$

Combining (5.11), (5.12) and (5.13) and using (3.5), we obtain

$$
\lim _{\substack{y \rightarrow 0 \\ y \in C^{\prime} \Subset C}}\langle f(z) / P(i z), \psi(x)\rangle=(2 \pi)^{n}\langle g(t), \phi(t)\rangle=\langle\mathscr{F}[g], \psi(x)\rangle,
$$

so that

$$
\begin{aligned}
\langle f(z), \psi(x)\rangle & =\langle f(z) / P(i z), P(i z) \psi(x)\rangle \rightarrow\langle\mathscr{F}[g], P(i x) \psi(x)\rangle \\
& =\langle P(i x) \mathscr{F}[g], \psi(x)\rangle
\end{aligned}
$$

as $y=\operatorname{Im}(z) \rightarrow 0, y \in C^{\prime} \Subset C$.

Since $U=(P(-D) g(t)) \in\left(S_{a_{k}}^{b_{q}}\right)^{\prime}, \mathscr{F}[U] \in\left(S_{b_{k}}^{a_{q}}\right)^{\prime}$. Let $\psi(x) \in S_{b_{k}}^{a_{4}}$; then there exists an element $\chi^{\iota}(t)=\chi(-t) \in S_{a_{k}}^{b_{a}}$ such that $\psi(x)=\mathscr{F}\left[\chi^{\iota}(t): x\right]$, and this $\chi^{\iota}(t)$ is given in terms of $\psi(x)$ by

$$
\chi^{-}(t)=\mathscr{F}^{-1}[\psi(x) ;-t]=(2 \pi)^{-n} \int_{\mathbf{R}^{n}} \psi(x) \exp (i\langle x, t\rangle) d x
$$


Hence by (3.5), Definition 3.3 and Lemma 3.4, we have

$$
\begin{aligned}
\langle\mathscr{F}[U], \psi(x)\rangle & =(2 \pi)^{n}\left\langle U, \chi^{\nu}(t)\right\rangle \\
& =\left\langle P(-D) g(t), \int_{\mathbf{R}^{n}} \psi(x) \exp (i\langle x, t\rangle) d x\right\rangle \\
& =\left\langle g(t), P(D) \int_{\mathbf{R}^{n}} \psi(x) \exp (i\langle x, t\rangle) d x\right\rangle \\
& =\left\langle g(t), \int_{\mathbf{R}^{n}} \psi(x) P(i x) \exp (i\langle x, t\rangle) d x\right\rangle .
\end{aligned}
$$

Since $\left\{a_{k}\right\} \in \mathfrak{M}$, by Lemma 3.7 , the multiplication by the polynomial $P(i x)$ is a continuous operation on $S_{h_{h}}^{a_{q}}$ for $\left|e_{\alpha}\right| \leqslant Q L^{|\alpha|} a_{|\alpha|}$. Thus $\psi(x) \in S_{h_{k}}^{a_{q}}$ implies $(P(i x) \psi(x)) \in S_{b_{k}}^{a_{q}}$, so there exists a function $\theta^{\nu}(t)=\theta(-t) \in S_{a_{k}}^{b_{q}}$ for which

$$
P(i x) \psi(x)=\mathscr{F}\left[\theta^{\vee}(t) ; x\right] \text { and } \theta^{\vee}(t)=\mathscr{F}^{-1}[P(i x) \psi(x) ;-t] \text {. }
$$

Thus from (5.16) and (3.5) we have

$$
\begin{aligned}
\langle\mathscr{F}[U], \psi(x)\rangle & =(2 \pi)^{n}\left\langle g(t), \theta^{\vee}(t)\right\rangle \\
& =\langle\mathscr{F}[g], P(i x) \psi(x)\rangle=\langle P(i x) \mathscr{F}[g], \psi(x)\rangle,
\end{aligned}
$$

which shows that $\mathscr{F}[U]=P(i x) \mathscr{F}[g] \in\left(S_{b_{k}}^{a}\right)^{\prime}$. Combining (5.15) and (5.17) we prove that $f(z) \rightarrow \mathscr{F}[U] \in\left(S_{b_{k}}^{a}\right)^{\prime}$, as $y=\operatorname{Im}(z) \rightarrow 0, y \in C^{\prime} \Subset C$, in the weak topology of $\left(S_{b_{k}}^{a_{q}}\right)^{\prime}$, as desired. This completes the proof of the theorem.

The space of functions $f(z)$ considered by De Roever [19, Theorem 2.21(ii)], restricted to cones under consideration in this section, is a subspace of the space $H_{C}^{h}$ defined in this paper. The function $f(z)$ considered by De Roever satisfies the growth condition (5.2) and is holomorphic in $\mathbf{R}^{n}+i C$, whereas in our case $f(z)$ satisfies (5.2) but is required to be holomorphic in $T\left(C^{\prime}, m\right)$ only, where $C^{\prime} \Subset C$. Furthermore, $\left(S_{b_{k}}^{a}\right)^{\prime} \subset Z_{M}^{\prime}$, the dual of $Z_{M}$, considered by De Roever [19], and the injection can easily be shown to be continuous. Thus Theorem 5.2 gives more precise information about the distributional boundary values of functions which are analytic in tubular radial domains and which satisfy the growth condition (5.2) than was given by [19, Theorem 2.21(ii)].

The following is a stronger theorem than just the converse to Theorem 5.2.

TheOREM 5.3. Assume $\left\{b_{k}\right\} \in \mathfrak{R}$. Let $C$ be an open, convex cone and let $U(h, C)$ be defined by (5.1). Let $g$ be a tempered ultradistribution with support in $U(h, C)$, which for $m>0$ can be represented as $g=P_{m}(D) G_{m}$, where $P_{m}(D)$ is an ultradifferentiable operator and $G_{m}$ is a continuous function on $\mathbf{R}^{n}$ which satisfies

$$
\left|G_{m}(\xi)\right| \leqslant K(m) \exp (m\|\xi\|) .
$$

Then the function $f(z) \stackrel{\Delta}{=} \mathscr{F}\left[\exp (-\langle t, y\rangle) g_{t}\right](x)$, whose boundary value equals $\mathscr{F}[g]$, satisfies: for every $m>0, C^{\prime} \Subset C$ and $L>0$ there is a constant $K=K\left(m, C^{\prime}, L\right)>0$ such that

$$
|f(z)| \leqslant K \exp \{b(L\|z\|)+h(y)+\sigma\|y\|\}, \quad y \in C^{\prime},\|y\| \geqslant m .
$$


Proof. From $\S 3$ we know that $g$ is an element of $\mathscr{D}_{b_{q}}^{\prime}$ and for such a $g$ we have the inequality [19, Theorem 2.24(ii), p. 61]

$$
|f(z)|=|\langle g, \exp (i\langle t, z\rangle)\rangle| \leqslant K \exp \{b(L\|z\|)+h(y)\}, \quad y \in C^{\prime},\|y\| \geqslant m .
$$

Since for any $\sigma>0$ and $y \in C^{\prime},\|y\| \geqslant m$, $\exp (\sigma\|y\|)>1$, we have the desired result.

6. The space $H_{C}^{0}$. In the special case $h(y)=0$, we shall show that more information can be obtained concerning the function $f(z) \in H_{C}^{0}$ than was given in Theorem 5.2. It will be shown that $f(z) \in H_{C}^{0}$ can be recovered as the Fourier-Laplace transform of the constructed distribution $U \in\left(S_{a_{k}}^{b_{a}}\right)^{\prime}$ and also as the Fourier transform in $\left(S_{b_{k}}^{a}\right)^{\prime}$ of $\exp (-\langle y, t\rangle) V_{t}$. We shall prove that $f(z)$ satisfies a strong boundedness property in $\left(S_{b_{k}}^{a}\right)^{\prime}$.

We now state without proof a lemma which is needed in the study of functions in $H_{C}^{0}$. This has been proved by Carmichael and Milton [7, Lemma 10].

LeMMA 6.1. Let $C$ be an open convex cone, and let $C^{\prime}$ be an arbitrary compact subcone of $C$. Let $g(t), t \in \mathbf{R}^{n}$, be a continuous function with support in $C^{*}=U(0, C)$. Let

$$
|g(t)| \leqslant M\left(C^{\prime}, m\right) \exp (\langle\zeta, t\rangle+\sigma|\zeta|) \quad\left(t \in \mathbf{R}^{n}\right),
$$

for all $\sigma>0$, where $M\left(C^{\prime}, m\right)$ is a constant depending on $C^{\prime} \subset C$ and $m$, and (6.1) is independent of $\zeta \in C^{\prime} \backslash\left(C^{\prime} \cap N(0, m)\right)$, where $m>0$ is arbitrary but fixed. Let $y$ be an arbitrary but fixed point of $C^{\prime} \backslash\left(C^{\prime} \cap N(0, m)\right)$. Then $\exp (-\langle y, t\rangle) g(t) \in L^{p}$, $1 \leqslant p<\infty$, as a function of $t \in \mathbf{R}^{n}$.

Lemma 6.2. Assume that $b_{k}$ satisfies (M.1). Let $C$ be an open convex cone and $C^{\prime}$ an arbitrary compact subcone of $C$. Let $U=P(D) g(t)$, where $P(D)$ is the ultradifferentiable operator defined by (3.9) and $g(t)$ is a continuous function on $\mathbf{R}^{n}$ satisfying (6.1). Let $\operatorname{supp}(U) \subseteq C^{*}=U(0, C)$. Then $f(z)=\langle U, \exp (i\langle z, t\rangle)\rangle$ is an element of $H_{C}^{0}$.

Proof. Let $C^{\prime}$ be an arbitrary compact subcone of $C$ and $m>0$ be arbitrary but fixed. $C^{*}$ is a regular set $\left[23\right.$, pp. 98, 99]; thus $\operatorname{supp}(g)=\operatorname{supp}(U) \subseteq C^{*}$. Consider the function

$$
f(z)=\langle U, \exp (i\langle z, t\rangle)\rangle=P(-i z) \int_{C^{*}} g(t) \exp (i\langle z, t\rangle) d t .
$$

Following the technique of Carmichael and Milton [7] it can now be shown that $f(z)$ is analytic for $z \in T\left(C^{\prime}, m\right), C^{\prime} \Subset C, m>0$ is arbitrary, and $|f(z)| \leqslant$ $K\left(C^{\prime}, m\right)|P(-i z)|$. Now, using inequality (3.11) and the fact that for any $\sigma>0$ and $y \in C^{\prime} \backslash\left(C^{\prime} \cap N(0, m)\right), \exp (\sigma\|y\|)>1$ we have

$$
|f(z)| \leqslant K^{\prime}\left(C^{\prime}, m\right) \exp (b(L\|z\|)+\sigma\|y\|) .
$$

TheOREM 6.3. Assume $\left\{a_{k}\right\},\left\{b_{k}\right\} \in \mathfrak{M}$. Let $f(z) \in H_{C}^{0}$ and let $C^{\prime}$ possessing property $\mathrm{P}$ be an arbitrary compact subcone of the open convex cone $C$. Then there exists a unique element $U \in\left(S_{a_{k}}^{h_{q}}\right)^{\prime}$ with $\operatorname{supp}(U) \subseteq U\left(0 ; C^{\prime}\right)=\{t:\langle t, y\rangle \geqslant 0, y \in$ $\left.C^{\prime}\right\}$ such that:
(i) $\exp (-\langle y, t\rangle) U_{t} \in\left(S_{a_{k}}^{b_{q}}\right)^{\prime}$ for all $y \in C$;
(ii) $f(z)=\langle U, \exp (i\langle z, t\rangle)\rangle\left(z \in T\left(C^{\prime}, m\right)\right)$; 
(iii) $f(z)=\mathscr{F}\left[\exp (-\langle y, t\rangle) U_{t}\right], z=x+i y \in T\left(C^{\prime}, m\right)$ in the sense of equality in $\left(S_{b_{k}}^{a}\right)^{\prime}$;

(iv) $\left\{f(z): y=\operatorname{Im}(z) \in C^{\prime} \backslash\left(C^{\prime} \cap N(0, m)\right),|y| \leqslant Q\right\}$ is a strongly bounded set in $\left(S_{b_{k}}^{a}\right)^{\prime}$, where $Q$ is arbitrary but fixed positive real number greater than $m$;

(v) $f(z) \rightarrow \mathscr{F}[U] \in\left(S_{b_{k}}^{a_{q}}\right)^{\prime}$ in the strong (weak) topology of $\left(S_{b_{k}}^{a_{q}}\right)^{\prime}$ as $y=\operatorname{Im}(z) \rightarrow 0$, $y \in C^{\prime} \Subset C$.

Proof. Theorem 5.2 ensures the existence of the unique element $U \in\left(S_{a_{k}}^{b_{a}}\right)^{\prime}$ with $\operatorname{supp}(U) \subseteq U\left(0, C^{\prime}\right)$ such that $f(z) \rightarrow \mathscr{F}[U] \in\left(S_{b_{k}}^{a_{q}}\right)^{\prime}$ in the weak topology of $\left(S_{b_{k}}^{a_{q}}\right)^{\prime}$ as $y \rightarrow 0, y \in C^{\prime} \Subset C$. From the proof of Theorem 5.2 we have $\exp (-\langle y, t\rangle) U_{t} \in$ $\left(S_{a_{k}}^{b_{q}}\right)^{\prime}, \forall y \in C$, and also $U=P(-D) g(t)$, where $P(D)$ is defined by (3.9) and $g(t)$ is a continuous function. From (5.5) and (5.7) it follows that

$$
|g(t)| \leqslant M\left(C^{\prime}, m\right) \exp (\langle y, t\rangle+\sigma\|y\|)
$$

for all $\sigma>0$ and all $y \in C^{\prime} \Subset C$. Therefore, by Lemma 6.2, $\langle U, \exp (i\langle z, t\rangle)\rangle$ exists as an analytic function of $z \in T\left(C^{\prime}, m\right)$. A simple calculation yields

$$
\langle U, \exp (i\langle z, t\rangle)\rangle=P(i z) \mathscr{F}[\exp (-\langle y, t\rangle) g(t): x] \quad\left(z \in T\left(C^{\prime}, m\right)\right) .
$$

In view of Lemma 6.1, the Fourier transform exists in the $L^{1}$, as well as the $L^{2}$, sense. Combining (5.8) and (6.2) we arrive at (ii).

Now we prove (iii). Let $\psi \in S_{b_{k}}^{a}$. Then there exists an element $\theta^{\vee}(t)=\theta(-t) \in S_{a_{k}}^{b_{q}}$ such that $\psi(x)=\mathscr{F}\left[\theta^{\triangleleft}(t) ; x\right]$ and $\theta^{\iota}(t)=\mathscr{F}^{-1}[\psi(x) ;-t]$. For $y$ an arbitrary but fixed point of $C^{\prime} \backslash\left(C^{\prime} \cap N(0, m)\right)$ we have

$$
\exp (-\langle y, t\rangle) \theta^{\sim}(t)=(2 \pi)^{-n} \int_{\mathbf{R}^{n}} \psi(x) \exp (i\langle z, t\rangle) d x \quad(z=x+i y),
$$

and $\exp (-\langle y, t\rangle) \theta^{\smile}(t) \in S_{a_{k}}^{b_{q}}$, since $\theta^{\smile}(t) \in S_{a_{k}}^{b_{q}}$. Further, since $f(z)=$ $\langle U, \exp (i\langle z, t\rangle)\rangle, f(z) \in\left(S_{b_{k}}^{\left.a_{q}\right)^{\prime}}\right.$ as a function of $x=\operatorname{Re}(z) \in \mathbf{R}^{n}$ for arbitrary but fixed $y \in C^{\prime} \backslash\left(C^{\prime} \cap N(0, m)\right)$. In view of (ii) and (6.3) we have

$$
\begin{aligned}
\langle f(z), \psi(x)\rangle & =\langle\langle P(-D) g(t), \exp (i\langle z, t\rangle)\rangle, \psi(x)\rangle \\
& =\int_{\mathbf{R}^{n}} P(i z) \psi(x) \int_{C^{*}} g(t) \exp (i\langle z, t\rangle) d t d x \\
& =\int_{C^{*}} g(t) \int_{\mathbf{R}^{n}} P(i z) \psi(x) \exp (i\langle z, t\rangle) d x d t \\
& =\left\langle U, \int_{\mathbf{R}^{n}} \psi(x) \exp (i\langle z, t\rangle) d x\right\rangle \\
& =(2 \pi)^{n}\left\langle\exp (-\langle y, t\rangle) U, \theta^{-}(t)\right\rangle .
\end{aligned}
$$

Since by (i), $\exp (-\langle y, t\rangle) U_{t} \in\left(S_{a_{k}}^{b_{a}}\right)^{\prime}$, the right-hand side is meaningful. In view of (3.5) this can be written as

$$
\langle f(z), \psi(x)\rangle=\langle\mathscr{F}[\exp (-\langle y, t\rangle) U], \psi(x)\rangle, \quad \psi \in S_{b_{k}^{a}}^{a_{q}} .
$$

This proves (iii). 
To prove (iv) assume $\Phi$ is a bounded set in $S_{a_{k}}^{b_{q}}$; then $\Phi^{\check{\nu}}=\{\check{\phi(t)}=\phi(-t)$ : $\phi \in \Phi\}$ is a bounded set in $S_{a_{k}}^{b_{a}}$. Let $\phi^{\swarrow} \in \Phi^{\swarrow}$. Since $\operatorname{supp}(U) \subseteq C^{*}$, we have

$$
\left\langle\exp (-\langle y, t\rangle) U, \phi^{\swarrow}(t)\right\rangle=\left\langle U, \lambda(t) \exp (-\langle y, t\rangle) \phi^{`}(t)\right\rangle,
$$

where $\lambda(t)$ is the same as defined by (4.7) in which $b$ is taken to be zero. By Lemma 4.6,

$$
\left\{\lambda(t) \exp (-\langle y, t\rangle) \phi^{`}(t): \phi \in \Phi, y \in C^{\prime} \backslash\left(C^{\prime} \cap N(0, m)\right),|y| \leqslant Q\right\}
$$

is a bounded set in $S_{a_{k}}^{b_{q}}$, where $Q$ is an arbitrary but fixed real number greater than $m$. Since $U \in\left(S_{a_{k}}^{b_{q}}\right)^{\prime}, U$ is continuous and bounded in $S_{a_{k}}^{b_{q}}$. Thus

$$
\begin{aligned}
& \left\{\left\langle\exp (-\langle y, t\rangle) U, \phi^{`}(t)\right\rangle: \phi \in \Phi, y \in C^{\prime} \backslash\left(C^{\prime} \cap N(0, m)\right),|y| \leqslant Q\right\} \\
& \quad=\left\{\left\langle U, \lambda(t) \exp (-\langle y, t\rangle) \phi^{`}(t)\right\rangle: \phi \in \Phi, y \in C^{\prime} \backslash\left(C^{\prime} \in N(0, m)\right),|y| \leqslant Q\right\}
\end{aligned}
$$

is a bounded set in the complex plane. Since $\Phi$ is an arbitrary bounded set in $S_{a_{k}}^{b_{a}}$, $\left\{\left(\exp (-\langle y, t\rangle) U_{t}: y \in C^{\prime} \backslash\left(C^{\prime} \cap N(0, m)\right),|y| \leqslant Q\right)\right\}$ is a strongly bounded set in $\left(S_{a_{k}}^{b_{q}}\right)^{\prime}$. Since the Fourier transform is a strongly continuous mapping of $\left(S_{a_{k}}^{b_{q}}\right)^{\prime}$ onto $\left(S_{b_{k}}^{a}\right)^{\prime}$, the set

$$
\begin{aligned}
\{f(z): y & \left.=\operatorname{Im}(z) \in C^{\prime} \backslash\left(C^{\prime} \cap N(0, m)\right),|y| \leqslant Q\right\} \\
& =\left\{\mathscr{F}\left[\exp (-\langle y, t\rangle) U_{t}: y=\operatorname{Im}(z) \in C^{\prime} \backslash\left(C^{\prime} \cap N(0, m)\right),|y| \leqslant Q\right]\right\}
\end{aligned}
$$

is strongly bounded in $\left(S_{b_{k}}^{a}\right)^{\prime}$.

It remains to prove (v). From the proof of (iv) we have

$$
\begin{aligned}
\left\langle\exp (-\langle y, t\rangle) U, \phi^{`}(t)\right\rangle & =\left\langle U, \lambda(t) \exp (-\langle y, t\rangle) \phi^{`}(t)\right\rangle \rightarrow\left\langle U, \lambda(t) \phi^{`}(t)\right\rangle \\
& =\left\langle U, \phi^{\check{(}(t)}\right\rangle
\end{aligned}
$$

by Lemma 4.5, as $y \rightarrow 0, y \in C^{\prime} \subset C$. Thus the sequence $\left\{\exp (-\langle y, t\rangle) U: y \in C^{\prime}\right.$ $\subset C$ \} converges in the weak topology of $\left(S_{a_{k}}^{b_{q}}\right)^{\prime}$ to $U \in\left(S_{a_{k}}^{b_{a}}\right)^{\prime}$ as $y \rightarrow 0, y \in C \subset C$. But from $\S 3, S_{a_{k}}^{b_{q}}$ is a Montel space; therefore by Edwards [10, Corollary 8.4.9, p. 510], $\left\{\exp (-\langle y, t\rangle) U: y \in C^{\prime} \subset C\right\}$ converges in the strong topology of $\left(S_{a_{k}}^{b_{a}}\right)^{\prime}$ to $U \in\left(S_{a_{k}}^{b_{a}}\right)^{\prime}$ as $y \rightarrow 0, y \in C^{\prime} \subset C$. Using the fact that the Fourier transform is strongly continuous from $\left(S_{a_{k}}^{b_{a}}\right)^{\prime}$ to $\left(S_{b_{k}}^{a_{q}}\right)^{\prime}$, we have from (6.4) that

$$
\left\{f(z): y=\operatorname{Im}(z) \in C^{\prime} \subset C\right\}=\left\{\mathscr{F}[\exp (-\langle y, t\rangle) U]: y=\operatorname{Im}(z) \in C^{\prime} \subset C\right\}
$$

converges in the strong topology of $\left(S_{b_{k}}^{a_{q}}\right)^{\prime}$ to $\mathscr{F}[U] \in\left(S_{b_{k}}^{a_{q}}\right)^{\prime}$ as $y \rightarrow 0, y \in C^{\prime} \subset C$. This completes the proof of the theorem.

Results converse to Theorems 5.2 and 6.3 are given in the following theorem.

TheOREM 6.4. Assume $\left\{a_{k}\right\},\left\{b_{k}\right\} \in \mathfrak{M}$. Let $C$ be an open convex cone such that each compact subcone $C^{\prime} \subset C$ satisfies property $P$. Let $U=P(-D) g(t)$, where $g(t)$ is a continuous function on $\mathbf{R}^{n}$ satisfying $(6.1)$ and $P(D)$ is the ultradifferential operator defined by (3.9). Let $\operatorname{supp}(U) \subseteq C^{*}=U(0, C)$. Then $U \in\left(S_{a_{k}}^{b_{a}}\right)^{\prime}$, and there exists a function $f(z) \in H_{C}^{0}$ satisfying (i)-(iv):

(i) $f(z)=\langle U, \exp (i\langle z, t\rangle)\rangle, z \in T\left(C^{\prime}, m\right), C^{\prime} \subset C$; 
(ii) $f(z)=\mathscr{F}\left[\exp (-\langle y, t\rangle) U_{t}\right], z \in T\left(C^{\prime}, m\right), C^{\prime} \subset C$, where the equality in (ii) is in the $\left(S_{b_{k}}^{a_{a}}\right)^{\prime}$ sense;

(iii) $\left\{f(z): y=\operatorname{Im}(z) \in C^{\prime} \backslash\left(C^{\prime} \cap N(0, m)\right),|y| \leqslant Q\right\}$ is a strongly bounded set in $\left(S_{b_{k}}^{a}\right)^{\prime}$, where $Q$ is an arbitrary but fixed real number greater than $m$;

(iv) $f(z) \rightarrow \mathscr{F}[U] \in\left(S_{b_{k}}^{a_{q}}\right)^{\prime}$ in the strong (weak) topology of $\left(S_{b_{k}}^{a_{a}}\right)^{\prime}$ as $y=\operatorname{Im}(z) \rightarrow 0$ $\left(y \in C^{\prime} \subset C\right)$.

Proof. Since $C^{*}$ is a regular set $[23,98,99], \operatorname{supp}(g)=\operatorname{supp}(U) \subseteq C^{*}$. Let $C^{\prime}$ be an arbitrary but compact subcone of $C$ and let $m>0$ be arbitrary. As in the proof of Theorem 5.2 it can be shown that $g(t) \in\left(S_{a_{k}}^{b_{q}}\right)^{\prime}$, and hence $U=P(-D) g(t) \in$ $\left(S_{a_{k}}^{b_{q}}\right)^{\prime}$. Therefore, by Lemma 6.2, $f(z)=\langle U, \exp (i\langle z, t\rangle)\rangle \in H_{C}^{0}$. For this $f(z)$ and $U \in\left(S_{a_{k}}^{b_{q}}\right)^{\prime}$, results (iii), (iv), (v) of Theorem 6.3 are true.

\section{Appendix.}

Equality of the spaces $S_{a_{k}}^{b_{q}}$ and $S_{a_{k}} \cap S^{b_{q}}$. Throughout this section we shall assume that $x$ is a one-dimensional real variable. Instead of assuming condition (M.1), we shall assume that $a_{k}$ satisfies: there is a constant $d_{1}>0$ such that

$$
\left(\begin{array}{l}
k \\
j
\end{array}\right) a_{k-j} a_{j} \leqslant d_{1} a_{k}, \quad 0 \leqslant j \leqslant k
$$

THEOREM A.1. Let $a_{k}$ satisfy (M.1) and (M.2), and $b_{k}$ satisfy (A.1) and (M.2). Then

$$
S_{a_{k}} \cap S^{b_{q}}=S_{a_{k}}^{b_{q}} \text {. }
$$

Proof. Evidently, $S_{a_{k}}^{b_{q}} \subset\left[S_{a_{k}} \cap S^{b_{q}}\right]$.

Now, let $\phi \in S_{a_{k}} \cap S^{b_{q}}$. Then there exist constants $C>0$ and $h>1$ such that for all $k \in \mathbf{N}, q \in \mathbf{N}$, we have

$$
\left|x^{k} \phi(x)\right| \leqslant C h^{k} a_{k} \text { and }\left|\phi^{q}(x)\right| \leqslant C h^{q} b_{q} .
$$

Using integration by parts, the Leibnitz product-derivative formula and inequalities (A.3) we have

$$
\begin{aligned}
\left\|x^{k} \phi^{q}\right\|_{2}^{2} & \leqslant \sum_{j=0}^{q}\left(\begin{array}{c}
q \\
j
\end{array}\right)\left(\begin{array}{c}
2 k \\
j
\end{array}\right) j ! \int_{-\infty}^{\infty}\left|x^{2 k-j} \overline{\phi(x)} \phi^{(2 q-j)}(x)\right| d x \\
& \leqslant \sum_{j=0}^{q}\left(\begin{array}{c}
q \\
j
\end{array}\right)\left(\begin{array}{c}
2 k \\
j
\end{array}\right) j !\left\|\left(1+x^{2}\right) x^{2 k-j} \phi(x)\right\|_{\infty}\left\|\phi^{(2 q-j)}(x)\right\|_{\infty} \int_{-\infty}^{\infty} \frac{d x}{1+x^{2}} .
\end{aligned}
$$

Next, using inequalities (M.1), (M.2) and (A.1), we easily get

$$
\left\|x^{k} \phi^{q}(x)\right\|_{2}^{2} \leqslant C L^{2(k+q)} a_{k}^{2} b_{q}^{2}, \quad L=h(1+\varepsilon), \varepsilon>0 .
$$

Now, in view of Lemma 4.1 and inequality (A.4), we have

$$
\begin{aligned}
\left\|x^{k} \phi^{q}(x)\right\|_{\infty} & \leqslant(2 \sqrt{\pi})^{-1}\left(\left\|x^{k} \phi^{q}(x)\right\|_{2}+\left\|x^{k} \phi^{(q+1)}(x)\right\|_{2}+\left\|k x^{k-1} \phi^{q}(x)\right\|_{2}\right) \\
& \leqslant(2 \sqrt{\pi})^{-1} C L^{k+q} a_{k} b_{q}\left(1+L b_{q+1} / b_{q}+L^{-1} k a_{k-1} / a_{k}\right) \\
& \leqslant(2 \sqrt{\pi})^{-1} C L^{k+q} a_{k} b_{q}\left(1+L K H^{q+1} b_{1}+L^{-1} d_{1} / a_{1}\right) \\
& \leqslant C_{1} L_{1}^{k+q} a_{k} b_{q}, \quad L_{1}=L(1+\delta), \delta>0 .
\end{aligned}
$$

Hence $\phi \in S_{a_{k}}^{b_{q}}$, and consequently $\left[S_{a_{k}} \cap S^{b_{q}}\right] \subset S_{a_{k}}^{h_{q}}$. This completes the proof. 
Note that the conditions of the above theorem are satisfied by $\left\{(p !)^{\alpha}\right\}, \alpha>1$.

Acknowledgements. The author is thankful to the referee for his helpful comments and suggestions. The work was partly supported by Research Grant No. Math. $1402 / 28$, Science Centre, King Saud University and by a grant from University Grants Commission, New Delhi.

\section{REFERENCES}

1. K. I. Babenko, On a new problem of quasi-analyticity and on the Fourier transformation of entire functions. Trudy Moskov. Mat. Obshch. 5 (1956), 523-542. (Russian)

2. A. Beurling, Quasi-analyticity and general distributions, Lectures 4 and 5, A.M.S. Summer Institute, Stanford 1961.

3. G. Björck, Linear partial differential operators and generalized distributions, Ark. Mat. 6 (1966). 351-407.

4. S. Bochner and W. T. Martin, Seteral complex variables, Princeton Univ. Press. Princeton. N. J., 1948 .

5. H. J. Bremermann, Distributions, complex variables, and Fourier transforms, Addison-Wesley, Reading, Mass.. 1965.

6. R. D. Carmichael, Distributional boundan values of analytic functions in tubular radial domains, Indiana Univ. Math. J. 20 (1971), 843-853.

7. R. D. Carmichael and E. O. Milton. Distributional boundan values in the dual spaces of spaces of type $\mathscr{S}$. Pacific J. Math. 56 (1975), 385-422.

8. F. Constantinescu. Analytic properties of nonstrictly localizable fields, J. Math. Phys. 12 (1971), 293-298.

9. F. Constantinescu and W. Thalheimer, Ultradistributions and quantum fields: Fourier Laplace transforms and boundary values of analytic functions, Rep. Math. Phys. 16 (1979), 167-180.

10. R. E. Edwards, Functional analysis, Holt, Rinehart and Winston. New York, 1965.

11. L. Ehrenpreis, Analytic functions and the Fourier transform of distributions. I, Ann. of Math. (2) 63 (1956), 129-159.

12. Analytic functions and the Fourier transform of distributions. II. Trans. Amer. Math. Soc. 89 (1958), 450-483

13. Analytically uniform spaces and some applications. Trans. Amer. Math. Soc. 101 (1961), $52-74$.

14. I. M. Gel'fand and G. E. Shilov, Generalized functions, Vol. II, Academic Press, New York, 1964.

15. A. Jaffe, High energy behaviour of strictly localizable fields, Phys. Rev. 158 (1967), 1454-1461.

16. H. Komatsu, Ultradistributions. I: Structure theorems and a characterization. J. Fac. Sci. Univ. Tokyo Sect. IA Math. 20 (1973), 25-105.

17. R. S. Pathak. Analytic functions having distributional boundary e'alues in $W^{\prime}$-spaces. Math. Proc. Cambridge Philos. Soc. 87 (1980), 227-242.

18. Cauchy and Poisson integral representations for ultradistributions of compact support and distributional boundary values, Proc. Roy. Soc. Edinburgh Sect. A 91 (1981), 49-62 and 96 (1984), 179.

19. J. W. De. Roever, Complex Fourier transformation and analytic functionals with unbounded careers, MC Tract. 89, Math. Centrum. Amsterdam, 1978.

20. J. W. De. Roever, Analytic representations and Fourier transforms of analytic functionals in $Z^{\prime}$ carried by the real space. SIAM J. Math. Anal. 9 (1978), 996-1019.

21. C. Roumieu, Sur quelques extensions de la notion de distribution. Ann. Sci. École Norm. Sup. (3) 77 (1960), 41-121.

22. Ultra-distributions definés sur $\mathbf{R}^{n}$ et sur certaines classes de cariètés differentiables. J. Analyse Math. 10 (1962/63), 153-192.

23. L. Schwartz. Theorie des distributions, 3rd ed., Hermann. Paris, 1966.

24. R. Streater and A. S. Wightman, PCT, spin and statistics and all that, Benjamin. New York. 1964.

25. H. G. Tillman, Darstellung der Schwartschen Distribution Durch analytische Funktionen. Math. Z. 77 (1961), 106-124.

26. F. Treves, Topological vector spaces, distributions and kernels, Academic Press. New York. 1967. 
27. V. S. Vladimirov, Methods of the theory of functions of several complex variables, M.I.T. Press, Cambridge, Mass., 1966.

28. D. Vogt and H. J. Petzsche, Representation of ultradistributions by boundary values of holomorphic functions (to appear).

29. A. H. Zemanian, Generalized integral transformations, Interscience, New York, 1968.

Department of Mathematics, Banaras Hindu University, Varanasi, India (Current address)

Department of Mathematics, King Saud University, Riyad, Saudi arabia 